TABLE II

CAvity Parameters for D-38 MM AND $\alpha=6.10^{-4} \mathrm{CM}^{-1}$ AT 100 Mtorr

\begin{tabular}{lccc}
\hline $\mathrm{N} / \mathrm{K}$ & $8 / 1$ & $12 / 1$ & $16 / 3$ \\
\hline $\mathrm{d}[\mathrm{m}]$ & 2.08 & 1.25 & 0.85 \\
$\mathrm{R}[\mathrm{m}]$ & 26.0 & 36.7 & 5.0 \\
$\mathrm{C}(\mathrm{F} 1 \mathrm{~g} .4)$ & $4.010^{-6}$ & $5.710^{-6}$ & $8.510^{-6}$ \\
$\mathrm{a}[\mathrm{mm}]\left(\mathrm{r}_{\mathrm{O}}=\frac{\mathrm{D}}{4}\right)$ & 5.5 & 10.8 & 2.25 \\
$\mathrm{P}_{\mathrm{abs}} / \mathrm{P}_{\mathrm{O}}[\%]$ & 77.05 & 70.66 & 64.39 \\
\hline
\end{tabular}

A similar approach can be made for rectangular waveguide FIR lasers with curved mirrors using pump beam traces in a plane. There is then no restriction in degeneracy values.

\section{OPEN RESONATORS}

It is worthwhile to reexamine here open FIR resonators. These offer single transverse mode operation together with lossless propagation and with reflections at the mirrors nearly free of coupling losses. There is no restriction to cavities with $d<R$ and small $d / R$ values. Because of the larger FIR mode diameter, smaller injection hole diameters result from (3), which again reduces FIR roundtrip losses. Thus, from the standpoint of pump efficiency and FIR cavity losses, open resonators are superior to waveguide cavities.

\section{CONCLUSIONS}

The importance of high degeneracy of the FIR cavity for an efficient use of the IR pump power has been outlined.
Such cavities also exhibit a low sensitivity to length adjustment because the separation of the resonance frequencies of neighboring transversal modes is proportional to $K / N$. The pump power reflected from such cavities and radiated back into the pump source is low. This reduces frequency pulling effects in the pump laser and the associated instabilities. A procedure to determine all the essential dimensions of highly degenerate FIR cavities except the laser tube diameter is given. The best choice for the tube diameter depends on a number of additional factors such as threshold pump intensity, desired FIR mode, FIR gain, and saturation parameter.

\section{REFERENCES}

[1] J. O. Henningsen and H. G. Jensen, "The optically pumped farinfrared laser: Rate equations and diagnostic experiments," IEEE J. Quantum Electron., vol. QE-11, pp. 248-252, June 1975.

[2] T. A. DeTemple and E. F. Danielewicz, "Con tinuous-wave $\mathrm{CH}_{3} \mathrm{~F}$ waveguide laser at $496 \mu \mathrm{m}$; Theory and experiment," IEEE $J$. Quantum Electron., vol. QE-12, pp. 40-47, Jan. 1976.

[3] M. Rosenbluh, R. J. Temkin and K. J. Button, "Submillimeter laser wavelength tables," Appl. Opt., vol. 15, pp. 2635-2644, Nov. 1976.

[4] I. A. Ramsay and J. J. Degnan, "A ray analysis of optical resonators formed by two spherical mirrors," Appl. Opt., vol. 9, pp. 385398, Feb. 1970

[5] D. C. Sinclair, Spectra-Physics, Laser Tech. Bull. 6, Apr. 1968.

[6] J. J. Degnan and D. R. Hall, "Finite-aperture waveguide-laser resonators," IEEE J. Quantum Electron., vol. QE-9, pp. 901-910, Sept. 1973.

[7] D. T. Hodges and J. R. Tucker, "Pump absorption and saturation in the $\mathrm{CH}_{3} \mathrm{~F}$ 496- $\mathrm{m}$ laser," Appl. Phys. Lett., vol. 27, pp. 667669 , Dec. 15,1975

[8] C. O. Weiss, "Pump saturation in molecular far-infrared lasers," IEEE J. Quantum Electron., vol. QE-12, pp. 580-584, Oct. 1976.

\title{
Quantum Counter for Far-Infrared Radiation
}

\author{
H. LENGFELLNER AND K. F. RENK
}

\begin{abstract}
Two types of three-level quantum counters, based on the their their application for up-conversion of far-infrared (FIR) radiation to inible light. It is reported on a tunable quantum counter of the inverted type: in optically pumped ruby, photons at $29 \mathrm{~cm}^{-1}$ are absorbed by the transition $\left.\bar{E}\left({ }^{2} E\right) \rightarrow 2 \overline{A(}{ }^{2} E\right)$ and lead to an increased $R_{2}$ the sescence radiation. By Zeeman splitting of the ${ }^{2} E$ levels, tuning of is detected frequency from $29 \mathrm{~cm}^{-1}$ to higher and lower frequencies possible (with $1 \mathrm{~cm}^{-1} /$ Tesla). Using the $891-\mathrm{GHz}$ radiation of an $\mathrm{HCN}$ laser we determine for the quantum counter a power conversion
\end{abstract}

\footnotetext{
Manuscript received November 19, 1976; revised December 28, 1976. The authors are with the Institut für Angewandte Physik, Universität Regensburg, Regensburg, Germany.
}

efficiency of the order of $10^{-6}$ and a frequency bandwidth of 360 MHz. We find the interesting result that phonon bottleneck due to resonant trapping of $29 \mathrm{~cm}^{-1}$ phonons can lead to an increase of the quantum efficiency by orders of magnitude.

\section{INTRODUCTION}

Q

UANTUM counters proposed by Bloembergen were realized for the detection of radiation in the near-infrared frequency region [1] and of $28-\mu$ laser radiation [2]. Indication of far-infrared (FIR) quantum counter action was found by Brown et al. [3] using for FIR absorption exchange coupled ground state levels of $\mathrm{Cr}^{3+}$ in dark ruby. 
In this paper we first discuss two principles of three-level quantum counters for the FIR. We then report on a tunable quantum counter which converts radiation of the $300-\mu \mathrm{m}$ wavelength region to visible light.

\section{Principles of FIR Quantum Counters}

Two different principles of three-level quantum counters are shown in Fig. 1(a) and (b). The scheme of Fig. 1(b) was first proposed by Bloembergen. The FIR radiation is absorbed from the ground state giving rise to occupation of the intermediate level 2 which leads to absorption of optical-pump radiation and occupation of level 3 . The occupation of level 3 is monitored by the optical signal radiation at the frequency $\nu_{31}$. In the alternative system of Fig. 1(a) the optical-pump radiation is absorbed from the ground state and ions are excited to level 2. The FIR radiation is absorbed by the transition $2 \rightarrow 3$. The occupation of level 3 is again monitored by the fluorescence radiation at $\nu_{31}$.

Here we want to point to one typical feature common to solid-state FIR quantum counters: the energy level which is occupied by the FIR absorption process has a very short relaxation time for spontaneous phonon emission due to the high density of states of the phonons in this frequency region. The relaxation time $T_{1}$ is in the order of $10^{-9}$ to $10^{-12} \mathrm{~s}$ dependent on the special system.

In the following we concentrate on the discussion of the quantum counter principle of Fig. 1(a). For the up-conversion power efficiency (fluorescent signal output power $P_{\text {out }}$ to FIR input power $P_{\text {in }}$ ) an upper limit is given by

$$
\eta_{\max }=\frac{T_{1}}{\tau} \frac{\nu_{31}}{\nu_{\mathrm{FIR}}},
$$

where it is assumed that the FIR radiation is completely absorbed by the transition $2 \rightarrow 3$. For typical values of $T_{1}=10^{-10} \mathrm{~s}, \tau=10^{-3} \mathrm{~s}$, and $\nu_{31} / \nu_{\mathrm{FIR}}=10^{3}$, we expect $\eta_{\max } \simeq 10^{-4}$. This value can be enlarged by the bottleneck factor $b$ if resonant phonons created by the relaxation process $3 \rightarrow 2$ are reabsorbed $b$ times. As will be shown (see Section III) $b$ can reach values higher than $10^{2}$.

In the more general case where the FIR radiation is not completely absorbed, the conversion efficiency has to be derived from the rate equations of the three-level system [compare Fig. 1(c)]. Under the influence of two radiation fields at frequencies $\nu_{12}$ and $\nu_{23}$ with energy densities $\rho_{12}$ and $\rho_{23}$, the levels 2 and 3 are excited with occupation numbers $N_{2}$ and $N_{3}$. If the system is not saturated the following rate equations hold:

$$
\begin{aligned}
& \dot{N}_{1}=-B_{12} \rho_{12} N_{1}+N_{2} \gamma_{21}+N_{3} \gamma_{31} \\
& \dot{N}_{2}=B_{12} \rho_{12} N_{1}-B_{23} \rho_{23} N_{2}+N_{3} \gamma_{32}-N_{2} \gamma_{21} \\
& \dot{N}_{3}=B_{23} \rho_{23} N_{2}-N_{3} \gamma_{32}-N_{3} \gamma_{31},
\end{aligned}
$$

where $B_{i j}$ are the Einstein coefficients for the optical transitions $i \rightarrow j$ and $\gamma_{i j}$ are the total transition rates for radiation transitions and radiationless transitions. In these rate equations thermal occupation processes are neglected; it is supposed that the system is cooled to low temperature.

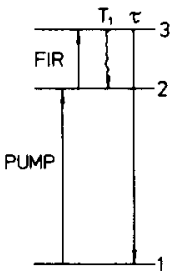

(a)

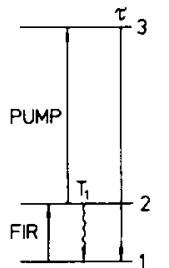

(b)

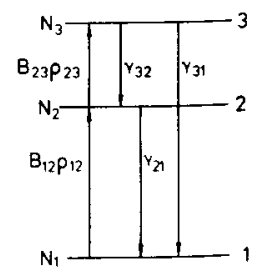

(c)
Fig. 1. Principles of three-level quantum counters. (a) The intermediate level 2 is populated by the pump radiation. Absorption of FIR quanta leads to occupation of level 3. (b) The FIR is absorbed by transitions from the ground state to the intermediate level. (c) A general scheme with the various transition probabilities is shown.

In the case of $\mathrm{CW}$-radiation fields, the time variations of the $N_{i}$ are zero and one obtains for the signal rate of the $3 \rightarrow 1$ transition the expression

$$
Z_{31}=N_{3} \gamma_{31} V=\frac{B_{12} \rho_{12} N_{1} B_{23} \rho_{23} V}{\left(\frac{\gamma_{32}}{\gamma_{31}}+1\right) \gamma_{21}+B_{23} \rho_{23}},
$$

where $V$ is the active volume of the quantum counter. Because $B_{23} \rho_{23}<<\gamma_{21}$ (weak FIR input) and $\gamma_{32}>>\gamma_{31}$, we obtain with the assumption $\gamma_{31} \simeq \gamma_{21}$ the signal rate

$$
Z_{31}=B_{12} \rho_{12} N_{1} B_{23} \rho_{23} T_{1} V \text {. }
$$

Equation (2) holds under the condition that the frequency bandwidths $\overline{\Delta \nu_{i j}}$ of the radiation fields $\rho_{i j}$ are larger than the widths $\Delta \nu_{i j}$ of the corresponding absorption lines. In experiments where narrow-band lasers are used to generate the pump and the FIR radiation $\left(\overline{\Delta \nu_{i j}}<\Delta \nu_{i j}\right)$ the transition probabilities $B_{i j} \rho_{i j}$ have to be changed for the pump radiation to $B_{12} \rho_{12}\left(\overline{\Delta \nu}_{12} / \Delta \nu_{12}\right)$ and for the FIR radiation to $B_{23} \rho_{23}\left(\overline{\Delta \nu_{23}}\right)$ $\left.\Delta \nu_{23}\right)$. In this case we obtain for the signal rate

$$
Z_{31}=B_{12} \rho_{12} \frac{\overline{\Delta \nu}_{12}}{\Delta \nu_{12}} N_{1} B_{23} \rho_{23} \frac{\overline{\Delta \nu}_{23}}{\Delta \nu_{23}} T_{1} V .
$$

The signal power leaving the quantum counter is given by

$$
P_{\text {out }}=Z_{31} h \nu_{31} \text {. }
$$

For the FIR power incident on the quantum counter we have the relation

$$
P_{\text {in }}=\rho_{23} \overline{\Delta \nu}_{23} c F_{\mathrm{FIR}},
$$

and, for the power of the pump radiation

$$
P_{p}=\rho_{12} \overline{\Delta \nu}_{12} c F_{p} .
$$

$F_{\mathrm{FIR}}$ and $F_{p}$ are the areas where FIR radiation and pump radiation pass through and $c$ is the speed of light in the quantum counter volume.

With the approximate relation for the (maximum) absorption coefficients,

$$
\alpha_{p}=\frac{h \nu_{12} B_{12} N_{1}}{c \Delta \nu_{12}}, \quad \text { for the pump radiation and },
$$

$\alpha_{\mathrm{FIR}}=\frac{h \nu_{23} B_{23} N_{2}}{c \Delta \nu_{23}}$, for the FIR radiation, 
We obtain for the quantum efficiency (with $\nu_{23}=\nu_{\text {FIR }}$ )

$$
\eta=\frac{P_{\text {out }}}{P_{\text {in }}}=\frac{\alpha_{p} l_{p} \alpha_{\text {FIR }} l_{\text {FIR }}}{N_{2}} \frac{T_{1}}{h \nu_{\text {FIR }}} \frac{P_{p}}{V},
$$

where $l_{\mathrm{FIR}}=\left(V / F_{\mathrm{FIR}}\right)$ is the path length of the FIR beam and $l_{p}=\left(V / F_{p}\right)$ the path length of the pump beam in the detector volume.

For the Fig. 1(a)-type quantum counter it is reasonable to write (3) in the form

$$
\eta=\alpha_{p} l_{p} l_{\mathrm{FIR}} \sigma_{\mathrm{FIR}} \frac{T_{1}}{h \nu_{\mathrm{FIR}}} \frac{P_{p}}{V}=l_{\mathrm{FIR}} \sigma_{\mathrm{FIR}} \frac{T_{1}}{h \nu_{\mathrm{FIR}}} \frac{P_{p}^{*}}{V} .
$$

As expected the efficiency increases proportionally to the absorbed pump power $P_{p}^{*}=\left(\alpha_{p} l_{p} P_{p}\right)$, and to the path length

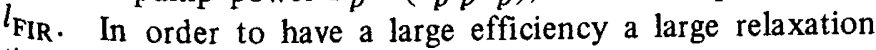
time $T_{1}$ and a large value of the FIR absorption cross section ${ }^{\sigma_{\text {FIR }}}=\left(\alpha_{\text {FIR }} / N_{2}\right)$ are desired.

For the system of Fig. 1(b) approximately the same expression for the power efficiency [see (3)] is obtained if realistic assumptions on the transition probabilities are made, especially, that the transition probability of the optical transition $3 \rightarrow 1$ is equal to or larger than that of the $3 \rightarrow 2$ transition. The efficiency can then be written

$$
\eta_{b}=\alpha_{\mathrm{FIR}} l_{\mathrm{FIR}} l_{p} \sigma_{p} \frac{T_{1}}{h \nu_{\mathrm{FIR}}} \frac{P_{p}}{V} .
$$

The efficiency increases linearly with pump power $P_{p}$ and path length $l_{p}$ of pump radiation. Because $\alpha_{\text {FIR }} l_{\text {FIR }} \simeq 1$ may be reached by suitable crystal doping for this quantum counter, a large absorption cross section $\sigma_{p}=\left(\alpha_{p} / N_{2}\right)$ for the optical pump radiation is desired (and again a long relaxation time $T_{1}$ ). Comparing the two quantum counter principles we find, for both types, comparable values for $\eta$ if the systems have comparable concentrations $N_{1}$ and similar transition properties $\left(\eta=\eta_{b}\right.$ if the product $\sigma_{\mathrm{FIR}} x \sigma_{p}$ is equal for the two systems). The values for $\eta$ can, however, be quite different, depending on the special system.

It should be noted that the quantum counter of Fig. 1(a) has a short response time given by the value $T_{1}$ of the relaxation process. For the other type, the response time is governed by the lifetime $\tau$ for the optical transition $3 \rightarrow 1$.

For the Fig. 1(a)-type quantum counter it is, however, more difficult to discriminate the signal at the frequency $\nu_{31}$ against the fluorescence radiation at the frequency $\nu_{21}$ than it is for the Fig. 1(b)-type.

\section{The Ruby Quantum Counter}

A quantum counter similar to that of Fig. 1(a) can be realized with ruby using the metastable $E$-levels as upper states. The principle is shown in Fig. 2. The ruby is pumped at low temperature with a mercury-xenon lamp and the $\bar{E}\left({ }^{2} E\right)$ level is effectively populated. Incoming FIR-quanta at $29 \mathrm{~cm}^{-1}$ are absorbed by the transition $\bar{E} \rightarrow 2 \bar{A}$ and give rise to an $R_{2}$. fluorescence signal.

In contrast to the scheme of Fig. 1(a) the pump radiation is not absorbed directly by the $1 \rightarrow 2$ transition, but indirectly via the pump bands of ruby. The quantum efficiency of this

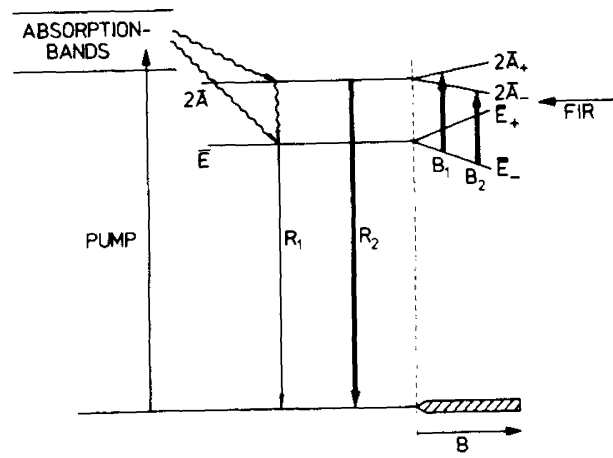

Fig. 2. Ruby FIR quantum counter. FIR photons are absorbed by the transition $E \rightarrow 2 A$ and cause an increase of $R_{2}$ fluorescence. The $\bar{E}$ level is populated by radiationless transitions from the absorption bands. In a magnetic field $B$ the excited state levels split according to the Landé factors $g(\bar{E})=2.445$ and $g(2 \bar{A})=1.47$.

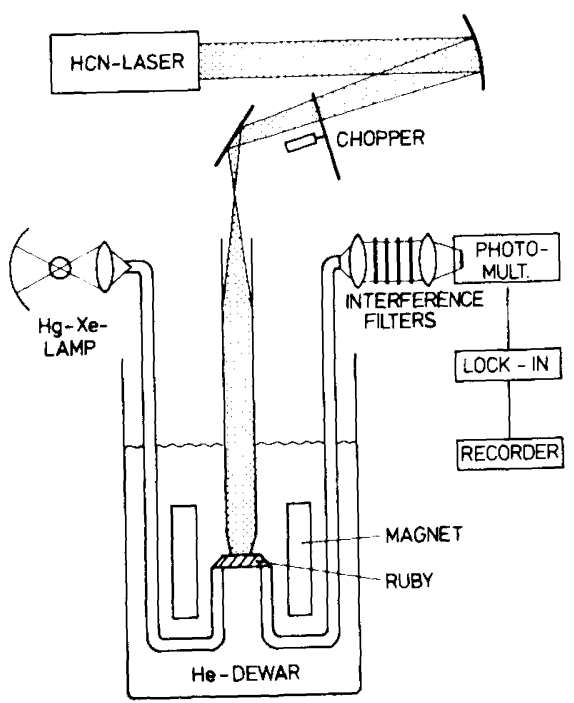

Fig. 3. Experimental arrangement of the FIR ruby quantum counter.

system is still described by (4) in which the value of $P_{p}^{*}$ is proportional to the optical-pump power.

The experimental arrangement is shown in Fig. 3. We used the chopped $891-\mathrm{GHz}$ radiation of an $\mathrm{HCN}$ laser for FIR source. With a magnetic field parallel to the $c$-axis of the crystal, Zeeman splitting of the levels $\bar{E}$ and $2 \bar{A}$ is obtained. For two magnetic fields the system can be tuned to resonance with the laser frequency as indicated in Fig. 2. The $R_{2}$ radiation is separated from the strong $R_{1}$-background with interference filters, detected with a photomultiplier and registrated using lock-in technique.

The quantum counter signal is shown in Fig. 4 for the $\bar{E}_{-} \rightarrow$ $2 \bar{A}_{+}$transition at the field $B_{1} \simeq 5 \mathrm{kG}$. From the signal halfwidth of $130 \mathrm{G}$ we obtain a bandwidth $\Delta \nu \simeq 360 \mathrm{MHz}$ $\left(0.012 \mathrm{~cm}^{-1}\right)$. A similar signal is obtained at $B_{2} \simeq 20 \mathrm{kG}$, which leads to the same detector bandwidth. We conclude a lifetime for the $2 \bar{A}$ level of $T_{1} \simeq(2 \pi \Delta \nu)^{-1}=4.4 \times 10^{-10} \mathrm{~s}$. This value is in good agreement with a theoretical estimate of $3 \times 10^{-10} \mathrm{~s}$ [4].

We have measured the dependence of the signal power $P_{\text {out }}$ as a function of the optical-pump power. The result is shown in Fig. 5. For the signal at $B_{2} \simeq 20 \mathrm{kG}\left(\bar{E}_{-} \rightarrow 2 \bar{A}_{-}\right)$our mea- 


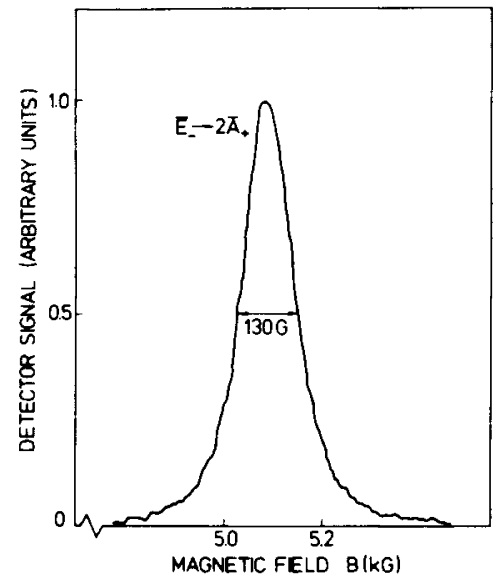

Fig. 4. Response of the ruby quantum counter in a magnetic field for the $891-\mathrm{GHz}$ radiation of an $\mathrm{HCN}$ laser.

surements indicate a linear relationship. For our highest pump power we estimate $P_{p}^{*} \simeq 0.1 \mathrm{~W}$. With this value we obtain from (4) for the power efficiency $\eta \simeq 10^{-6}$ with respect to the transition $\bar{E}_{-} \rightarrow 2 \bar{A}_{-}$.

For the signal at the magnetic field $B_{1}$ we find the interesting result that the signal power $P_{\text {out }}$ increases quadratically with the optical-pump power. This indicates a strong phonon bottleneck: [5] for the relaxation of the $2 A_{+}$level. The effective relaxation time is $T_{b}=T_{1}(1+b) \simeq b T_{1}$ where $b$ is the number of reabsorption processes for the resonant $29 \mathrm{~cm}^{-1}$ phonons. Replacing $T_{1}$ in (4) by $T_{b}$ and, regarding that $b$ increases proportionally to the concentration of excited $\mathrm{Cr}^{3+}$ ions we find that (for constant FIR input power) the signal output varies quadratically with the pump power, in agreement with our experimental curve. We conclude from our results that $b>100$ for our largest optical-pump power. Our result demonstrates that the bottleneck effect can increase the efficiency of the quantum detector by orders of magnitude.

For low optical pump powers (see Fig. 5) the quantum counter signal for the $\bar{E}_{-} \rightarrow 2 \bar{A}_{+}$transition is at least two orders of magnitude smaller than that for the $\bar{E}_{-} \rightarrow 2 \bar{A}_{-}$transition. This indicates a very small absorption cross section for the $\bar{E}_{-} \rightarrow 2 \bar{A}_{+}$transition.

The sensitivity of our present quantum counter is noiselimited by the $R_{2}$ occupation arising from the pump process which leads to an occupation of the $2 \bar{A}$ level given by $N_{3}=$ $\left(\gamma_{31} / \gamma_{32}\right) \times N_{2} \simeq 10^{-7}$. If the $R_{1}$ level would be directly pumped by a dye-laser this problem would vanish and the intrinsic noise of the quantum counter should disappear as the temperature is low enough. We estimate that with a $\mathrm{CW}$ dye-laser emitting $0.1 \mathrm{~W}$ at the $R_{1}$ line a minimum signal in the order of $10^{-11} \mathrm{~W}$ can be detected. In this estimate it is assumed that the noise is caused by the photomultiplier with about 50 dark counts per second. The strong $R_{1}$ radiation has to be suppressed by a factor of $10^{16}$ with interference filters for which a total transmission of $10^{-3}$ for the $R_{2}$ radia-

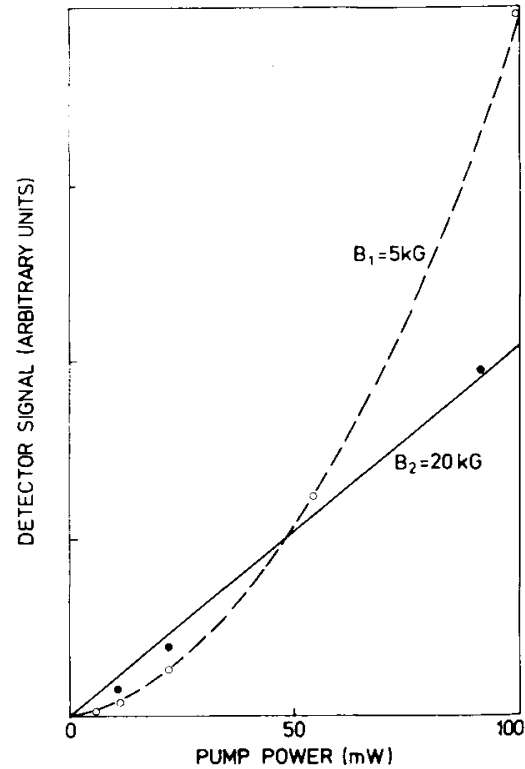

Fig. 5. Quantum counter signal as a function of the absorbed pump power $P_{p}^{*}$.

tion is assumed. An additional increase in the sensitivity is expected by a multiple pass arrangement for the FIR, thus effectively enlarging $l_{\text {FIR }}$ in (4).

The Zeeman splitting of the $\bar{E}$ and $2 \bar{A}$ levels can be used to tune the FIR frequency at which the quantum counter responds. Dependent on the Zeeman components, the detector can be tuned from $29 \mathrm{~cm}^{-1}$ to higher and lower energies with about $1 \mathrm{~cm}^{-1} / \mathrm{T}$.

\section{ACKNOWLEDGMENT}

The authors would like to thank A. Szabo for helpful discussions.

\section{REFERENCES}

[1] M. R. Brown and W. A. Shand, "The quantum counter," in Advances in Quantum Electronics, vol. 1, D. W. Goodwin, Ed. New York: Academic, 1970, pp. 2-75.

L. Esterowitz, J. Schnitzler, J. Noonan, and J. Bahler, "Rare earth infrared quantum counter," Appl. Opt., vol. 7, pp. 2053-2070, Oct. 1968.

K. G. Sewell and W. B. Volz, "Direct infrared image up-conversion with a praseodymium chloride quantum counter," Appl. Phys. Lett., vol. 23, pp. 104-106, July 15, 1973.

[2] M. Gundersen, "Conversion of $28-\mu$ far-infrared radiation to visible light using bound excitons in CdS," Appl. Phys. Lett., vol. 24, pp. 591-592, June 15, 1974.

[3] M. R. Brown and R. J. Strain, "Quantum-counting spectroscopy," J. Appl. Phys., vol. 37, pp. 4806-4810, Dec. 1966.

[4] M. Blume, R. Orbach, A. Kiel, and S. Geschwind, "Spin-lattice relaxation in the $E\left({ }^{2} E\right)$ state of $d^{3}$ ions in corundum," Phys. Rev. vol. 139, pp. A314-A321, July 5, 1965.

[5] S. Geschwind, G. E. Devlin, R. L. Cohen, and S. R. Chinn, "Orbach relaxation and hyperfine structure in the excited $\bar{E}\left({ }^{2} E\right)$ state 0 , $\mathrm{Cr}^{3+}$ in $\mathrm{Al}_{2} \mathrm{O}_{3}$," Phys. Rev., vol. 137, pp. A1087-A1100, Feb. 15 1965.

K. F. Renk and J. Deisenhofer, "Imprisonment of resonant pho" nons observed with a new technique for the detection of $10^{12-112}$. phonons," Phys. Rev. Lett., vol. 26, pp. 764-766, Mar. 29, 